\section{S01.2 BACTERIAL VAGINOSIS, VAGINAL MICROBIOTA, AND FECUNDABILITY}

E Lokken*. University of Washington, Seattle, USA

10.1136/sextrans-2021-sti.20

Bacterial vaginosis (BV), high diversity vaginal microbiota, and presence of specific BV-associated bacteria have been associated with an increased risk of reproductive outcomes including pelvic inflammatory disease, miscarriage, and preterm birth. The effects of BV and vaginal microbiota on fecundity and fertility in the context of non-medically assisted reproduction is not yet fully characterized. In this session, we will summarize what is known about the relationship between vaginal microbiota disruption and fertility among infertile populations undergoing in-vitro fertilization, discuss results from a recently published study assessing BV and fecundability - the per menstrual cycle probability of pregnancy - among Kenyan women trying to conceive, and identify key considerations for future studies of how the vaginal microbiota may influence conception, implantation, and early pregnancy.

\section{SO1.3 NEW INSIGHTS INTO GARDNERELLA AND OTHER BV- ASSOCIATED BACTERIA}

N Cerca*. University of Minho, Braga, Portugal

10.1136/sextrans-2021-sti.21

Bacterial vaginosis (BV), the most common vaginal disorder in women of reproductive age, remains one of the most intriguing and controversial challenges in modern-day clinical microbiology. BV is associated with increased transmission of HIV and other STIs, and is characterized by the loss of normal vaginal flora and overgrowth of anaerobes such as Gardnerella. However, it is not clear which is the preceding step for BV development: the loss of resident lactobacilli that eventually allows the overgrowth of already resident Gardnerella species, or the colonization of Gardnerella, presumably acquired due to sexual transmission, which will lead to the displacement of vaginal lactobacilli. However, Gardnerella colonization does not always lead to BV. Importantly, a recent large genome sequencing study demonstrated that what was previously described as G. vaginalis are, in fact, distinct species of the genus Gardnerella. It has been hypothesized that the distinct Gardnerella species have different virulence potential and that this can explain why some Gardnerella are present in healthy women without developing BV.

While Gardnerella has been proposed as pivotal agents for BV development, other species are also found in the typical multi-species biofilms associated with BV. What is not known, so far, is if these other species are playing an active role in $\mathrm{BV}$ development or if are just a consequence of the lactobacilli depletion that occurs in the early stages of BV. In recent years, my research team has been working on elucidating how different bacterial species associated with BV might interact synergistically and enhance the polymicrobial biofilm observed in vivo. Our in vitro data has shown that (i) some species enhance Gardnerella mediated biofilms and (ii) different multispecies biofilm morphotypes are formed, with distinct tridimensional structures, which, in some cases, can explain why antimicrobial therapy often fails, resulting in very high rates of recurrent BV.

\section{S01.4 IMMUNE ACTIVATION BY THE GUT MICROBIOME IN HIV POSITIVE AND NEGATIVE MEN WHO HAVE SEX WITH MEN; POTENTIAL IMPLICATIONS FOR TRANSMISSION AND DISEASE PATHOGENESIS}

C Lozupone*, B Palmer, C Neff, E Yamada, S Li, T Campbell. University of Colorado, Anschutz Medical Campus, Aurora, USA

\subsection{6/sextrans-2021-sti.22}

Men who have sex with men (MSM) have differences in immune activation and gut microbiome composition compared with men who have sex with women (MSW). Gut microbiome differences have also been associated with HIV itself. Understanding whether gut microbiome composition impacts immune activation in HIV-negative and HIV-positive MSM has important implications since immune activation has been associated with HIV acquisition risk and disease progression. We have used both in vitro assays and gnotobiotic mouse models to show that MSM and HIV-associated gut microbiota increases immune activation, including $\mathrm{T}$ cell activation and expression of the HIV co-receptor CCR5. To investigate whether these findings could have implications for HIV replication, we infected primary human lamina propria cells stimulated with isolated fecal microbiota, and found that microbiota from MSM stimulated higher frequencies of HIV-infected cells than microbiota from MSW. We have also been mechanistically exploring the potential role of key correlated bacteria in both driving and suppressing gut $\mathrm{T}$ cell activation. This work has the potential to assist in efforts to reduce new HIV infections within the disproportionally affected population of MSM and to reduce chronic immune activation in this population.

\section{S02.1 ANTIMICROBIAL RESISTANCE AND EXTRAGENITAL STIS - PHARMACOKINETIC CONSIDERATIONS}

F Kong *. The University of Melbourne, Melbourne, Australia

\subsection{6/sextrans-2021-sti.23}

Rates of bacterial sexually transmitted infections (STIs) continue to rise, demanding treatments to be highly effective. However, curing infections faces significant challenges due to antimicrobial resistance for organisms such as Neisseria gonorrhoeae and especially at extragenital sites, such as for oropharyngeal gonorrhoeal infections. As few new treatments are entering the market, clinicians must optimize current available antibiotics, but robust data are lacking on how the antibiotic's properties or pharmacokinetics can be used to inform STI treatment regimens to improve treatment outcomes. This presentation discusses how factors related to the drug (e.g. penetration into tissue/fluids/cells, protein binding) and human (e.g. $\mathrm{pH}$, site of infection, immune response) can affect treatment outcomes. The presentation will focus in oropharyngeal gonorrhoeal infections. It discusses the potential methods for measuring antibiotic concentration in novel oropharyngeal samples other than blood including saliva and gingival crevicular fluid and the role tissue $\mathrm{pH}$ may have on 
treatment efficacy. It also highlights the sub-optimal efficacies of emerging treatments currently in clinical trial to treat extragenital gonorrhoeal infections and suggest optimisation during clinical trials could potentially improve the efficacy of these treatments to reach the marketplace while balancing patients side effects.

\section{SO2.3 MOLECULAR TESTING FOR STI ANTIMICROBIAL RESISTANCE: TACKLING A MOVING TARGET}

D Whiley*. The University of Queensland, Brisbane, Australia

10.1136/sextrans-2021-sti.24

The gonococcus is now presenting some serious public health and clinical challenges. Notably, antibiotic-resistant Neisseria gonorrhoeae strains continue to emerge and spread and now threaten key gonorrhoea treatments, including ceftriaxone. Alternate treatment strategies are needed. Individualized therapy, whereby a rapid resistance test is used to inform individual patient treatment, is one proposed strategy. The use of individualised therapy in now already embedded in treatment of antibiotic (azithromycin)-resistant Mycoplasma genitalium infection, but is still in its infancy for the gonococcus. Key challenges exist, including knowledge gaps relating resistance mechanisms, the fact that sequence targets may change over time. These hurdles are not insurmountable, and can potentially be overcome via enhanced international collaboration and sequence/data sharing.

\section{SO2.4 CONCORDANCE BETWEEN GENOTYPIC AND PHENOTYPIC RESISTANCE, AND IMPLICATIONS FOR SURVEILLANCE OF AMR}

M Cole*. Public Health England, UK

10.1136/sextrans-2021-sti.25

Culture-based phenotypic antimicrobial susceptibility testing (AST) is the gold-standard method for determining Neisseria gonorrhoeae antimicrobial resistance (AMR). AMR surveillance can be enhanced by the molecular detection of resistance directly from clinical specimens, usually by nucleic-acid amplification techniques (NAATs). AMR surveillance is then possible in areas that struggle with obtaining viable cultures. NAAT AMR detection can be more sensitive and quicker than culture-based AST and can subsequently improve sample size and representativeness. Available NAATs do not however reliably detect all resistance markers, so some selection is required.

Whole-genome sequencing (WGS) is normally performed using cultured isolates and this enables detection of all known resistance markers. If the sequence is linked to culture, then the data can be used to detect unknown resistance determinants. Additional advantages of using WGS for AMR surveillance activities include the identification of AMR clones and transmission networks to further enhance $\mathrm{N}$. gonorrhoeae molecular epidemiology.

Genotypic and phenotypic resistance concordance is high for some antimicrobials such as ciprofloxacin, so using genotypic, in place of phenotypic methods, can be done with confidence. For some antimicrobials, such as cefixime and ceftriaxone, resistance can be multi-factorial so multiple loci detection/sequencing and appropriate algorithms need to be applied to predict resistance. Even then, the predicted resistance may not always correlate with the MIC. Azithromycin can also be a challenge as the exact mechanisms of resistance have not been identified for all isolates.

As genotypic methods alone cannot detect the emergence of novel resistance and not all resistance mechanisms are known, then appropriate sampling would be required if NAATs or WGS were to be solely used in surveillance studies. It is likely that AST via phenotypic methods has a role in AMR surveillance for some time to come, but AMR surveillance would certainly be enhanced by genotypic resistance detection.

\section{S03.1 ROLE OF MYCOPLASMA GENITALIUM IN SYMPTOMS AND SYNDROMES IN CISGENDER WOMEN}

M Trent*. Johns Hopkins Schools of Medicine and Public Health, Baltimore, Maryland, USA

\subsection{6/sextrans-2021-sti.26}

Mycoplasma genitalium (MG) has been a known pathogen affecting reproductive health outcomes since the 1980s. However, it was not officially considered in the Centers for Disease Control and Prevention (CDC) STI treatment guidelines until 2015. The paucity of population-based epidemiological data and clinical outcomes data from randomized controlled trials limits the scope of the CDC recommendations. While increased attention to $\mathrm{MG}$ as a pathogen has been driven by complicated presentations of urethritis in cisgender males, MG also has significant implications for sexual and reproductive health outcomes in cisgender women, including maternal-child health outcomes. Recurrent and persistent infection may be associated with ongoing inflammation in the female genital tract that may increase secondary STI and HIV infection risk due to resulting shifts in vagina microbiota. While there are no public health control programs in the United States for MG, several other countries have recognized the importance of prioritizing MG as a public health issue. In this session, we will examine MG in the context of adolescent and young adult cisgender women's health disparities and discuss the potential for strategic interventions in practice.

\section{S03.2 WHAT IS THE ROLE OF QUINOLONE RESISTANCE TESTING IN THE MANAGEMENT OF M. GENITALIUM?}

G Murray*. Royal Women's Hospital, Melbourne, Australia

\subsection{6/sextrans-2021-sti.27}

In the wake of escalating macrolide resistance, resistance to fluoroquinolones has emerged as a problem requiring urgent attention. The mechanisms of resistance for macrolides (single nucleotide mutations in the $23 \mathrm{~S}$ rRNA gene) are well defined both in vitro and clinically through analysis of treatment failure; resistance-guided therapy based on these mutations can achieve first line cure levels of over 90\%. This contrasts with our understanding of the mechansims of fluoroquinolone resistance, where multiple single nucleotide polymorphisms in the parC gene have been postulated to contribute, but evidence supporting a role of each mutation is limited. Currently 